\title{
SUPERCONDUCTING RADIOFREQUENCY LINAC DEVELOPMENT AT FERMILAB ${ }^{*}$
}

\author{
STEPHEN D. HOLMES \\ Fermi National Accelerator Laboratory, P.O Box 500 \\ Batavia, IL, 60510, USA
}

\begin{abstract}
As the Fermilab Tevatron Collider program draws to a close, a strategy has emerged of an experimental program built around the high intensity frontier. The centerpiece of this program is a superconducting $\mathrm{H}^{-}$linac that will support world leading programs in long baseline neutrino experimentation and the study of rare processes. Based on technology shared with the International Linear Collider, Project $\mathrm{X}$ will provide multi-MW beams at 60-120 GeV from the Main Injector, simultaneous with very high intensity beams at lower energies. Project X also supports development of a Muon Collider as a future facility at the energy frontier.
\end{abstract}

\section{Strategic Context and Evolution of the Fermilab Complex}

Fermilab currently operates both the highest energy particle collider and the highest intensity accelerator-based neutrino beam in the world. This situation will change by the end of 2009 when the Large Hadron Collider (LHC) at CERN in Geneva, Switzerland will initiate operations at energies substantially higher than the Tevatron, and the J-PARC facility in Tokai, Japan, will initiate a long baseline neutrino program in strong competition with the Fermilab program. In anticipation of these developments the U.S. elementary particle physics (EPP) community has adopted a strategic plan for the U.S. over the coming decade [1] emphasizing forefront research on three frontiers: the energy frontier, the intensity frontier, and the cosmic frontier. This strategy includes accelerator-based initiatives on the energy and intensity, based both in the U.S. and abroad. The strategy recognizes that over the coming decade Fermilab will be the sole site for accelerator-based EPP in the U.S. The Fermilab strategy [2] is fully aligned with the U.S. plan, and features the development of a multiMegawatt proton source as the centerpiece of the long term U.S. program.

* Work supported by the Fermi Research Alliance, under contract to the U.S. Department of Energy 


\subsection{Evolution of the Fermilab Accelerator Complex}

Project $\mathrm{X}$ is the name applied to a multi-MW proton facility proposed for construction at Fermilab. Project $\mathrm{X}$ is based on an $\mathrm{H}^{-}$linear accelerator (linac) employing superconducting rf technology. Project $\mathrm{X}$ is the linchpin for future development of the Fermilab accelerator complex, providing long-term opportunities on both the energy and intensity frontiers. The technology development is closely aligned with the technologies required for the International Linear Collider (ILC), and this development preserves Fermilab as a possible host, or major contributor, to such a future facility. The development of multi-MW capabilities also could provide a basis for a future Muon Collider or Neutrino Factory. In parallel Project $X$ provides great flexibility on the intensity frontier, offering the opportunity for a long-term world-leading program in neutrino physics and other beyond-the-standard-model phenomena. Finally, the technology developed for Project $\mathrm{X}$ opens opportunities beyond traditional EPP applications, for example in cold-neutron physics and accelerator driven (subcritical) systems (ADS) for energy generation and/or transmutation of waste.

\section{Project $X$ Performance Criteria and Initial Configurations}

The initial design criteria for a multi-MW proton source have been established based on requirements outlined in the P5 report [1]:

- A neutrino beam for long baseline neutrino oscillation experiments based on a proton source capable of delivering at least $2 \mathrm{MW}$ of beam power at any energy over the range $60-120 \mathrm{GeV}$;

- High intensity $8 \mathrm{GeV}$ protons supporting muon and kaon precision experiments, simultaneous with the neutrino program; and

- A path toward a muon source for a possible future Neutrino Factory or Muon Collider. This requires that the initial facility retain the potential for upgrading to 2-4 MW at $8 \mathrm{GeV}$.

Two configurations that meet these requirements have been developed. The first is based on an $8 \mathrm{GeV}$ pulsed linac, and the second on a $2 \mathrm{GeV}$ continuous wave (CW) linac followed by an $8 \mathrm{GeV}$ rapid cycling synchrotron (RCS). Both configurations utilize the existing Recycler and Main Injector rings for the accumulation of beam and acceleration to $60-120 \mathrm{GeV}$. 


\subsection{Project $X$ Initial Configuration - 1}

An intial configuration, identified as Initial Configuration - 1 (IC-1), based on an $8 \mathrm{GeV}$ pulsed linac is shown schematically in Figure 1. This schematic corresponds to operations at $120 \mathrm{GeV}$; operations at $60 \mathrm{GeV}$ rely on a cycle time of roughly half that of $120 \mathrm{GeV}$, in order to preserve the total beam power without changing the number of protons accelerated per pulse.

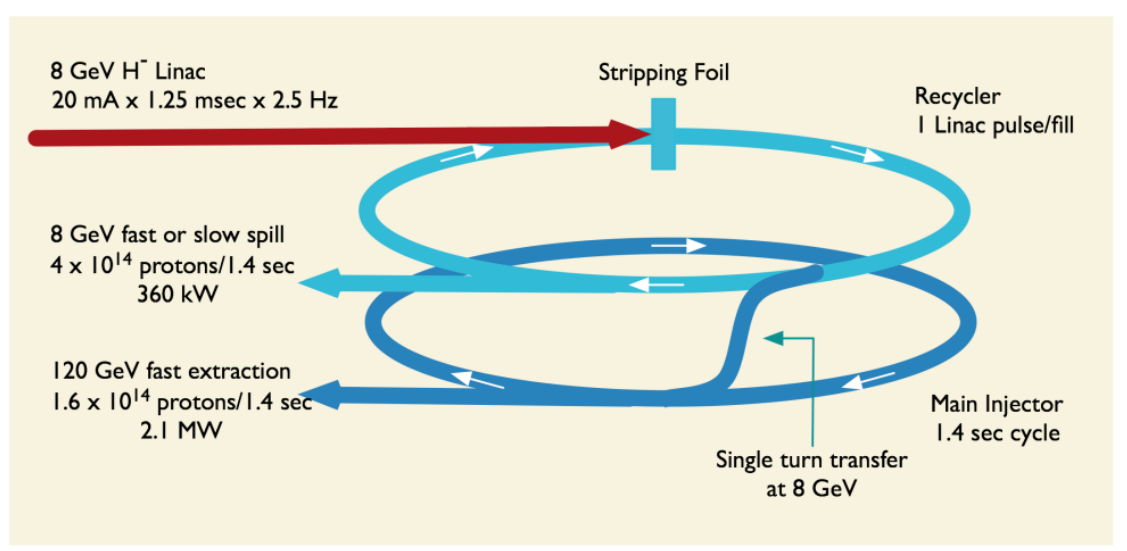

Figure 1. Project X Initial Configuration - 1 .

IC-1 is based on an $8 \mathrm{GeV}$, superconducting, pulsed $\mathrm{H}^{-}$linac. The linac beam is accumulated in the Recycler Ring over 100 turns, and is then transferred in a single turn to the Main Injector ring for acceleration and delivery to a neutrino target. The linac delivers $1.6 \times 10^{14} \mathrm{H}^{-}$per pulse at a $2.5 \mathrm{~Hz}$ repetition rate: the total beam power available at $8 \mathrm{GeV}$ is thus $500 \mathrm{~kW}$. The Main Injector operates on a 1.4 second cycle time at $120 \mathrm{GeV}$, corresponding to $2.1 \mathrm{MW}$ of beam power delivered to a neutrino program. In this scenario the Main Injector utilizes only two out of every seven linac pulses, leaving 5/7 of the linac pulses (360 $\mathrm{kW}$ at $8 \mathrm{GeV}$ ) available for other programs. In short, IC-1 is capable of delivering 2.1 MW at $120 \mathrm{GeV}$, simultaneous with $360 \mathrm{~kW}$ at $8 \mathrm{GeV}$.

Unfortunately IC-1 has limitations in providing a flexible platform for mounting a rare processes program - these programs (muons and kaons) typically require high duty factor beams that are traditionally provided by resonant extraction from a circular ring. The Recycler, because of its large ratio of circumference to energy is not well suited to resonant extraction - space charge effects and the 
need to create a substantial momentum spread to stabilize the beam compromise the overall efficiency of the process. Other smaller rings at Fermilab may be better suited, most notably the Debuncher Ring with its larger aperture and smaller circumference. However, even in this ring simulations show that there is an upper limit to the time averaged beam power that can be resonantly extracted at about $150 \mathrm{~kW}$. We believe that a limit in this range is fundamental to the resonant extraction process, due to losses generated on a finite width extraction septum. Hence, we have explored options for providing the required beam structure, at very high power, without resort to resonant extraction.

\subsection{Project X Initial Configuration - 2}

Based on considerations described above with regard to the rare processes program a second configuration, Initial Configuration - 2 (IC-2), has been developed. IC-2, shown schematically in Figure 2, is based on a $2 \mathrm{GeV} \mathrm{CW}$ linac operating at $1 \mathrm{~mA}$ average current. This configuration meets the same design criteria as IC-1, but significantly exceeds IC-1 in the performance for the rare processes program. Beam performance parameters for IC-2 are given in Table 1.

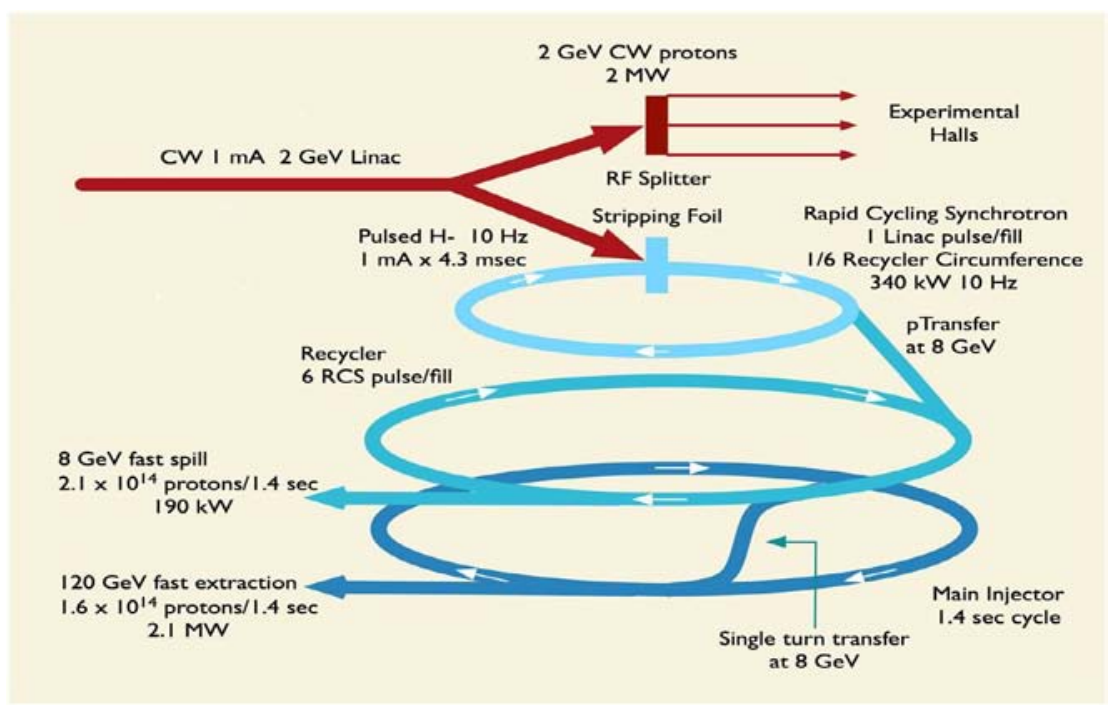

Figure 2. Project X Initial Configuration - 2. 
Table 1. Beam performance parameters for Project $\mathrm{X}$ Initial Configuration -2 .

\begin{tabular}{llr}
\hline Linac & & \\
Particle Type & $\mathrm{H}^{-}$ & \\
Kinetic Energy & 2.0 & $\mathrm{GeV}$ \\
Average Beam Current & 1.0 & $\mathrm{~mA}$ \\
Pulse Rate & $\mathrm{CW}$ & $\mathrm{Hz}$ \\
$\quad$ Beam Power & 2000 & $\mathrm{~kW}$ \\
$\quad$ Beam Power to 2 GeV Program & 1920 & $\mathrm{~kW}$ \\
RCS & & \\
$\quad$ Particle Type & protons & \\
Kinetic Energy & 8 & $\mathrm{GeV}$ \\
Cycle Time & 0.1 & $\mathrm{sec}$ \\
Particle per Pulse to Recycler & $2.6 \times 10^{13}$ & \\
Beam Power to 8 GeV Program & 200 & $\mathrm{~kW}$ \\
Main Injector/Recycler & & \\
Kinetic Energy (maximum) & 120 & $\mathrm{GeV}$ \\
Cycle Time & 1.4 & $\mathrm{sec}$ \\
Particles per Pulse & $1.6 \times 10^{14}$ & \\
Beam Power to 120 GeV & 2100 & $\mathrm{~kW}$ \\
\hline
\end{tabular}

In IC-2 the neutrino program is supported by the acceleration of beam in a rapid cycling synchrotron (RCS) that injects into the Recycler. The RCS is one sixth the circumference of the Recycler (and the Main Injector) and operates at $10 \mathrm{~Hz}$. The Main Injector operates at $120 \mathrm{GeV}$ on the same 1.4 second cycle as in IC-1. The RCS is filled every 0.1 seconds by diverting 4.3 msec of linac beam and injecting over 2000 turns. Six such cycles are then accumulated in the Recycler Ring and transferred to the Main Injector in a single turn every 1.4 seconds. 
With RCS operations at $10 \mathrm{~Hz}$ this leaves 8 of every 14 cycles available for supplying a fast extracted beam at $8 \mathrm{GeV}$ for other programs. In this mode of operation only $4 \%$ of the linac beam is diverted to the RCS, leaving nearly the full 2 MW available for a low energy rare processes program. Thus, IC-2 provides simultaneously, $1.9 \mathrm{MW}$ at $2 \mathrm{GeV}, 200 \mathrm{~kW}$ at $8 \mathrm{GeV}$, and $2.1 \mathrm{MW}$ at $120 \mathrm{GeV}$.

The rare processes program is supported directly from the CW linac with a bunch structure that can be individually tailored to the needs of the experimental program. The bunch format is created by a chopper at the front end with a bandwidth of $\sim 300 \mathrm{MHz}$. The ultimate destination of the bunches is determined by their phase with respect to a subharmonic (1/4) transverse deflector at the end of the linac that can send individual bunches to one of three different experimental areas, depending upon the phase of the bunch. The chopper must create bunch patterns that average to $1 \mathrm{~mA}$ over the $\sim 1 \mu \mathrm{sec}$ memory time of the superconducting cavities, but otherwise the disposition of current within the bunch stream is arbitrary.

An example is shown in Figure 3. Rf buckets are available at a frequency of 162.5 MHz for acceptance of beam particles. Bunches colored blue have a phase that will transmit them undeflected through the transverse deflector at the end of the linac. The red bunches carry a phase that will deflect them in one direction, and the green bunches in the opposite direction. In this particular example the blue bunches could be used for a muon-to-electron conversion experiment requiring beam on for 100 nsec followed by $\sim 1 \mu \mathrm{sec}$ of no beam. The red and green beams are both continuous. The average current over the cycle shown in the figure is $1 \mathrm{~mA}$.

Both IC-1 and IC-2 have been documented and are available online [3]. 


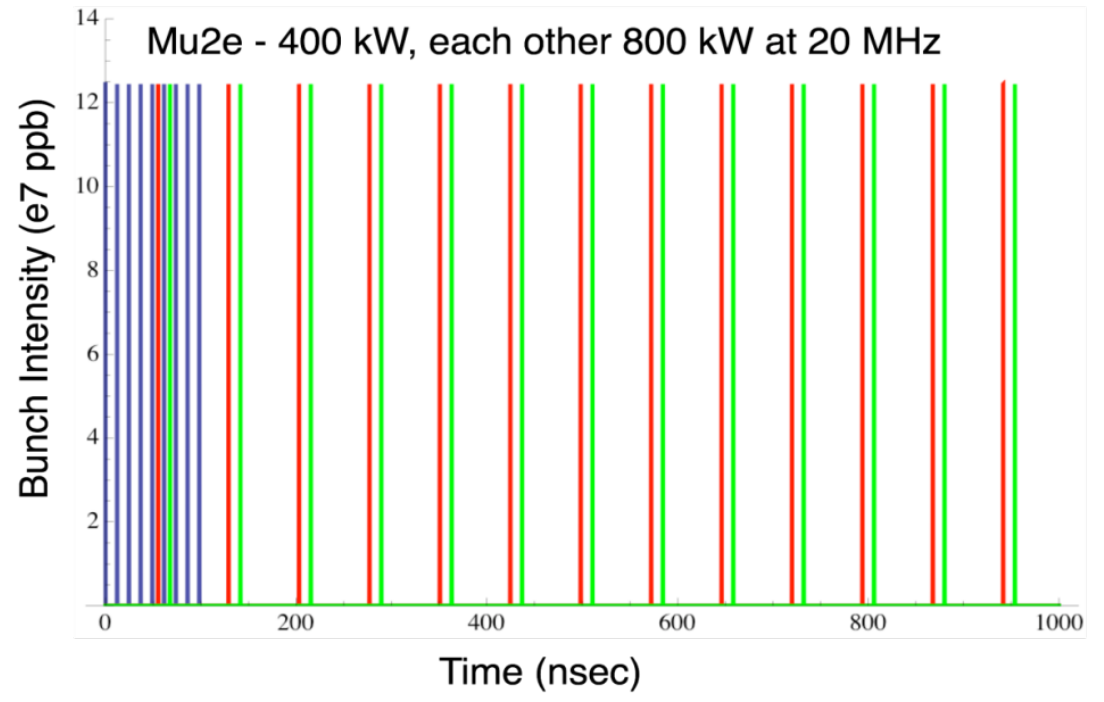

Figure 3. Possible bunch pattern averaging to $1 \mathrm{~mA}$ current, and capable of supplying $400 \mathrm{~kW}$ to one experiment, simultaneous with $800 \mathrm{~kW}$ to each of two additional experiments.

\section{Project X Research, Design, and Development Plan}

The goal of the Project X Research, Design, and Development (RD\&D) Plan is to complete a fully developed technical description, construction schedule, and cost estimate by 2013. This effort is currently underway and is being undertaken by a multi-institutional U.S. collaboration composed of Argonne National Laboratory, Brookhaven National Laboratory, Cornell University, Fermilab, Lawrence Berkeley National Laboratory, Michigan State University, Oak Ridge National Laboratory, Thomas Jefferson National Accelerator Facility, SLAC National Accelerator Laboratory, and the Americas Regional Team of the ILC Global Design Effort. Assuming successful completion of the RD\&D phase, physical construction could start in 2015, with construction completed over a roughly five year construction period.

\subsection{Technology Development}

Technology deployment for the IC-2 linac is shown in Figure 4. This configuration is not yet optimized and hence only represents an initial concept. 


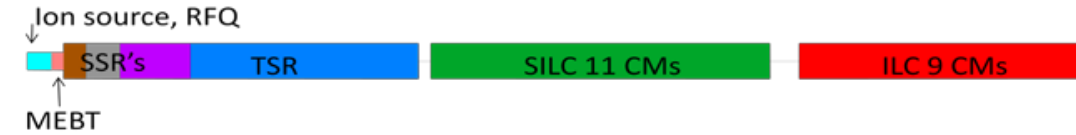

Figure 4. Initial configuration of the cw linac in IC-2.

A $10 \mathrm{~mA}$ dc ion source and $162.5 \mathrm{MHz}$ RFQ are followed by a MEBT operating at $2.5 \mathrm{MeV}$. Development of the flexible chopper described above, which resides in the MEBT, will be one of the most challenging goals of the RD\&D phase of the program. The upstream acceleration section is based on superconducting spoke resonator cavities designed to various betas and utilizing triple spoke resonators (TSR's) at the end. This section operates at $325 \mathrm{MHz}$ and is envisioned as extending up to roughly $450 \mathrm{MeV}$. The following section is based on squeezed elliptical cavities at roughly beta $=0.8$, and operating at 1.3 GHz. The technology in this section is "ILC like". Eleven cryomodules are envisioned in this section, taking the beam up to about $1.2 \mathrm{GeV}$. These cavities are operated with a gradient of $14 \mathrm{MV} / \mathrm{m}$. The linac is completed with nine beta $=1.0$ cyromodules, again operating at $1.3 \mathrm{GHz}$. This section takes the energy to $2.0 \mathrm{GeV}$, or possibly beyond. The cavity operating gradient in this section is $17 \mathrm{MV} / \mathrm{m}$ with a $\mathrm{Q}_{0}$ of $1.5 \times 10^{10}$. The cavity technology is very similar to that required for the ILC, although the couplers and rf distribution system will be different. We know that the deployment outlined here is not optimal and expect to modify the various beta and frequency transition points over the next several months. Prototypes of both the lowest beta single spoke resonator and the beta $=1.0$ elliptical cavities have been built and successfully tested.

\section{Other Applications}

The technology being developed for Project $\mathrm{X}$ is broadly applicable in areas beyond support of research in elementary particle physics. Examples of additional applications include:

- Accelerator Driven Energy Systems

- Rare isotope production for nuclear physics

- Neutron Sources

- X-ray FELs

- Energy recovery linacs

- Muon facilities for materials research... 
Several of these applications are discussed in detail in this workshop.

\section{Summary}

Project $\mathrm{X}$ is central to Fermilab's strategy for future development of the accelerator complex. Project $\mathrm{X}$ technology development is aligned with the needs of future Energy Frontier facilities such as the ILC and a Muon Collider. Construction of Project $\mathrm{X}$ would provide a world leading Intensity Frontier facility based on programs in neutrinos and rare processes.

Two intial configurations have been established that meet the general design criteria established for the facility. Both configurations support the delivery of 2 MW to a neutrino target, simultaneous with high power beam deliver to a low energy rare processes program. However the configuration based on a CW linac appears to offer significant advantages in terms of supporting the rare processes program, with an opportunity for multi-MW of beam power devoted to this program in parallel with the neutrino program.

Strong synergies exist with applications beyond elementary particle physics, either through the technology development or through the development of experimental programs based at the Project X facility. Such applications include, but are not limited to, Accelerator Driven Energy Systems, cold neutron sources, and other nuclear physics applications.

With sufficient support, Project X could be constructed over the time period 2015-1019.

\section{References}

1. Particle Physics Project Prioritization Panel, "U.S. Particle Physics: Scientific Opportunities, A Strategic Plan for the Next Ten Years”, May 2008,

http://www.science.doe.gov/hep/files/pdfs/P5_Report\%2006022008.pdf

2. Fermilab Steering Group Report, September 2007, http://www.fnal.gov/pub/directorate/steering/index.shtml

3. Project X website, http://projectx.fnal.gov/ 